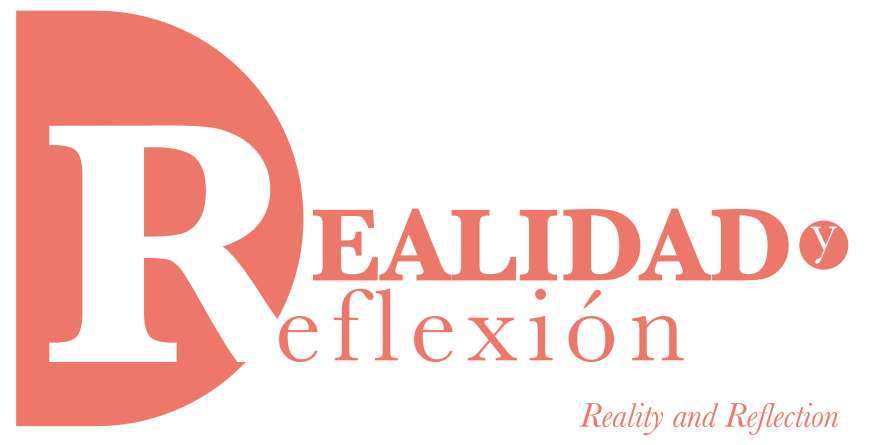

ISSN 1992-6510

e-ISSN 2520-9299

Año 19, N 49, San Salvador, El Salvador, Centroamérica. Revista Semestral Enero-Junio 2019

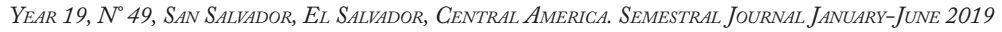

\title{
Metodología de diagnóstico logístico de almacenes y centros de distribución
}

\section{Diagnostic methodology for warehouses and distribution centers operations}

\section{RESUMEN}

Existen muchas metodologías de análisis para el mejoramiento de almacenes y centros de distribución. Este artículo expone, a grandes trazos, los componentes utilizados por la empresa Portal Logístico para desarrollar sus proyectos. Independientemente de la tecnología utilizada, los factores o componentes que se analizan son: análisis de infraestructura y áreas; procesos, inventarios, seguridad laboral y ocupacional; e indicadores logísticos o KPI.

Palabras clave: logística; cadena de suministro; centro de distribución; almacén; El Salvador.

ABSTRACT

There are many analytical methodologies for the improvement of warehouses and distribution centers operations. This article exposes the components used by a logistical consulting company to develop their projects. Regardless of the technology used, the factors that are analyzed are: infrastructure analysis and areas; Processes, inventories, occupational safety and logistical indicators (KPI)

Keywords: logistics, supply chain, warehouse, distribution center, El Salvador. 


\section{Introducción}

Es muy común encontrar que los profesionales y practicantes en logística aborden el tema del mejoramiento de sus almacenes o centros de distribución desde ópticas muy variadas y, por lo general, con enfoques no integrados. De hecho, los proyectos de mejoramiento y optimización de operaciones se complica pues ante el dilema de por dónde comenzar el análisis o qué herramientas utilizar, muchos dudan y se realizan análisis superficiales del tema. Al final, sin llegar realmente a las causas del pobre desempeño logístico.

Por esa razón, se da a conocer una metodología aplicable al diagnóstico y mejoramiento de centros de distribución con las características propias de las economías centroamericanas (escasa inversión tecnológica, infraestructura inapropiada, personal poco capacitado y sistemas informáticos fragmentados).

Tanto las empresas privadas como las instituciones gubernamentales requieren un uso racional de los recursos limitados (inventarios, capital humano, equipos, espacio y recursos económicos). Bien sea en la administración de medicamentos, insumos industriales, productos perecederos, electrónicos, telas, alimentos, bebidas, productos ferreteros, etc., no sólo importa mantener niveles de inventario óptimos, sino también mantener sus propiedades en buen estado y asegurarse de que el trabajador realiza sus labores en ambientes seguros. Esta es la función social más evidente del artículo. Dar unas luces en la oscuridad y contribuir a un uso más racional de los recursos.

\section{Información básica}

En este artículo se presenta una metodología lo suficientemente sencilla que se identificará con el acrónimo IPISI (Infraestructura, Procesos, Inventarios, Seguridad Laboral e Indicadores). Se buscan integrar los aspectos claves a analizar para el desarrollo de proyectos de mejoramiento de operaciones en centros de distribución.

\section{Pregunta de investigación}

¿Qué pasos deberían considerarse en el diagnóstico de las operaciones logísticas de un almacén o centro de distribución para mejorar su productividad?

\section{Justificación}

Con frecuencia, los profesionales a cargo de los almacenes y centros de distribución se pierden en los detalles al momento de realizar un diagnóstico de sus operaciones. Se enfocan en aspectos no esenciales o el alcance que se utiliza es muy limitado. Reconozco que esto ocurre tanto a profesionales jóvenes como a experimentados, ya que la productividad es un fenómeno complejo y multifactorial.

Proponemos un método sencillo basado en la experiencia de consultoría en el área para poder encarar estos proyectos de forma integral y responsable.

\section{La metodología}

La metodología IPISI con la que se analizan los almacenes y centros de distribución permitirá establecer estrategias integrales de mejora de la productividad del mismo. En ese sentido, pueden 
existir dos grandes grupos: metodologías cualitativas y cuantitativas.

Sorprende que pese al avance tecnológico y formación en el área, no todas las empresas manejan sistemas confiables de información y carecen de indicadores de desempeño de sus operaciones, es preciso combinar aspectos cualitativos. Para citar un caso en concreto, una compañía distribuidora de productos farmacéuticos, al no medir el porcentaje de nivel de servicio y no determinar las ventas diarias perdidas, no se había percatado que dejaba de facturar en promedio cinco mil dólares diarios (más de millón y medio de dólares al año). Hubo que calcular a partir de muestras representativas, ya que los sistemas informáticos no podían ofrecer esta información.

Igual encontraremos limitantes en cuanto a la información económica disponible porque muchas empresas prefieren ocultar costos a sus empleados en el área logística por razones de confiabilidad. ¿Se puede trabajar sin ellos? Desde luego que sí, aunque en algunos tópicos, principalmente inventarios, nos llevaría a conclusiones erróneas. Tómese el caso del análisis $\mathrm{ABC}$, antigüedad del inventario, valor del inventario, valoración de costos logísticos, etc.

La metodología IPISI, de igual forma, puede ser comparativa (analiza), descriptiva (expone) o normativa (valora). No es lo mismo buscar alternativas a un problema específico que buscar problemas o causa raíz de estos.

Es parte de la habilidad del consultor el adoptar unas y desechar otras. El contexto, la industria $y$ las situaciones particulares de negocio son variables que no se pueden ignorar. Ejemplo, las Buenas Prácticas de Manufactura BPM en la industria farmacéutica, las normativa de Análisis de Peligros y Puntos Críticos de Control APPCC o HACCP, etc.

\section{Factores de complejidad de los centros de distribución}

El punto de partida es reconocer dónde radica la complejidad de la operación en un almacén o centro de distribución. Saber su papel estratégico para conocer realmente cómo se genera la riqueza en la organización. Los almacenes y centros de distribución no son lo mismo. Estos últimos tienen una infraestructura diseñada para realizar las operaciones en forma eficiente, con mucha velocidad. Usualmente, manejan equipos y software que requieren una mayor instrucción o preparación académica de sus empleados. Lo importante es distribuir, no almacenar.

Los centros de distribución pueden agrupar almacenes (warehouses) que son infraestructuras que proveen un adecuado ambiente para guardar temporalmente bienes y materiales que requieran protección de los elementos. Deben ser diseñados para acomodar los arribos de los materiales a ser almacenados; el equipo de manejo de materiales; las áreas de recepción y despacho y sus parqueos asociados; y las necesidades requeridas por el personal.

La complejidad que se observa en los centros de distribución es muy variada y depende de una combinación de factores interdependientes (figura 1). Empresas de una misma industria pueden hacer su logística de formas muy diferentes aunque el mercado sea el mismo. 
El enfoque que tenga la gerencia sobre si se planifica a corto o largo plazo con un enfoque moderno y estratégico permitirá también resolver los dilemas de inversión apropiadamente. No es sólo cuestión de dinero, sino del enfoque hacia una lógica de desarrollo y crecimiento. Qué tan fácil es cambiar o replantearse los paradigmas actuales.
En buena parte, las empresas operan en localidades que no fueron construidas para negocio, no cuentan con sistemas informáticos robustos $y$, normalmente, como efecto de la rotación de personal, existe un conocimiento logístico deficiente.
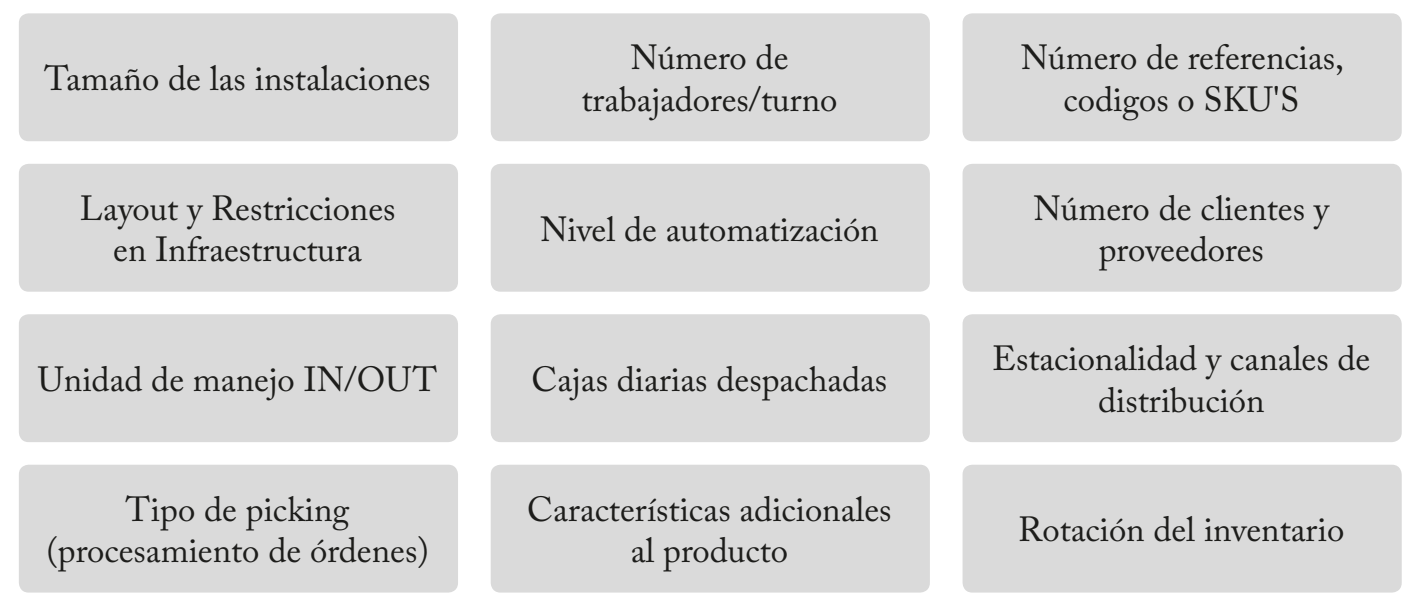

Figura $n .^{\circ} 1$. Factores de Complejidad de un Centro de Distribución. Fuente: elaboración propia.

Sin llegar a ser exhaustivos, los factores de complejidad que se detallan en la Figura n. 1 revisten importancia capital pues son estos los que pueden modificarse a discreción de la empresa para desarrollar estrategias logísticas diferenciadas y flexibles. El tamaño de las instalaciones, el número de trabajadores, variedad de productos, rotación del inventario, turnos requeridos, etc. Cuando no existe un adecuado balance y racionalidad en el uso de los factores, se generan cuellos de botellas y diversos problemas en la productividad de las operaciones.

Existen, desde luego, factores coyunturales y heredados como causa raíz de los problemas.
Para guardar enfoque y claridad en el análisis, las empresas deberían, al menos, analizar los componentes del modelo del autor del artículo (Figura n. ${ }^{\circ}$ 2) y que es utilizado frecuentemente por la consultora Portal Logístico en la práctica.

Sólo al finalizar el análisis de estos pilares fundamentales o fases, se tendrá una visión integral de lo que pasa en la realidad y se podrán reconocer e identificar las oportunidades de mejora que pueden implementarse. De acuerdo a planes y prioridades, las inversiones y cambios requeridos son decisiones en entornos complejos. Se recomienda conocer las prácticas que utilizan otras empresas del sector e incluso, 
- Análisis de Infraestructura y Areas

FASE 2 - Análisis de procesos

\section{- Análisis de Inventarios}

FASE 4 - Seguridad laboral y ocupacional

FASE5 Indicadores logísticos

Figura n. $^{\circ}$ 2. Fases de la metodología IPISI utilizada por Consultora Portal Logístico. Fuente: elaboración propia.

si se puede, visitar empresas extranjeras y congresos internacionales especializados para observar el funcionamiento de equipos, software, y otros.

Se asume como premisa el deseo sincero de la empresa u organización de mejorar su rendimiento con recursos propios, sin tomar en cuenta el outsourcing o alternativas de subcontratación de operadores logísticos. Aunque dicha estrategia del outsourcing puede ayudar a desarrollar seriamente todo el potencial de la empresa y ampliar sus capacidades, este punto merece un análisis particular en otro artículo.

\section{Componentes de la metodología}

\section{A. Fase 1: análisis de infraestructura y áreas}

La localización y la infraestructura utilizada ejercen una enorme influencia sobre el desempeño de las operaciones logísticas (recibo, acomodo, almacenamiento, preparación de pedidos $\mathrm{o}$ picking, despacho, croosdocking y otros).

Tómese el caso de aquel negocio que nació en una zona residencial y luego fue creciendo mediante la compra de casas aledañas que fueron reacomodándose paulatinamente como áreas de almacenes o bodegas. Sus operaciones, desde luego, van a verse impactadas por la distribución física de los productos, independientemente de los sistemas tecnológicos y de la capacidad del personal. Es una condición preexistente.

$\mathrm{El}$ alquiler de instalaciones debe hacerse con un enfoque de diseño nuevo y plantear modificaciones previas a su uso ya que será muy difícil trabajar mientras se realicen operaciones en ella. El alquiler debe revisar la idoneidad de las áreas de recibo, 
almacenamiento, despacho y espacios de maniobra suficientes para las unidades de transporte.

Otra realidad totalmente diferente puede significar el diseño de un almacén desde cero. Tómese en cuenta la finalidad del almacén, los volúmenes y flujos de productos, las operaciones que se realizarán y las características propias de los productos. Para tal efecto, los libros Facilities Planning, de James A. Tomkins, y el de Diseño de Instalaciones Industriales, de Stephan Konz, profundizan adecuadamente esta situación. La página web de Whole Business Design Guide WBDG (http://www.wbdg.org/ building-types/warehouse) provee también recomendaciones valiosas y estándares que se siguen en los Estados Unidos.

El análisis debe abarcar tanto los elementos constructivos como los de operación. Debe tomarse en cuenta el perfil del centro de distribución (cómo está la empresa en los factores planteados en la Figura n. ${ }^{\circ} 1$ ), de los flujos de operación y, por supuesto, de la naturaleza de los productos.

Las limitaciones constructivas (postes, paredes, calles, tuberías, ubicación de muelles de carga y descarga, etc.) dificultan la fluidez de la logística. Es necesario elaborar un layout o un plano de la distribución física, como en la figura n. ${ }^{\circ} 4$, para visualizarlas con claridad.

La ubicación de artículos en un almacén no es aleatoria. Debe planearse en función de la rotación del producto. Primero deben establecerse las zonas ABC, luego las categorías, sectores o departamentos (como las que existen en una ferretería o supermercado - ver Figura n. 4) y, finalmente, los productos. Los casos especiales de artículos de alto valor, peligrosos o voluminosos deber tratarse como excepciones.

El análisis de la infraestructura permite darle continuidad a las operaciones y mantener al mínimo las reparaciones y modificaciones en el tiempo. Los siguientes cinco puntos aclararán su alcance.

\section{1) Calidad del suelo y piso:}

Antes de construir o modificar la estructura existente, debe elaborarse un análisis de suelo que permita determinar si la construcción es técnicamente viable. Pueden existir cárcavas, suelos arcillosos o mucha humedad, lo cual representan grandes riesgos. Posteriormente, en caso de viabilidad, debe realizarse la construcción del piso utilizando hormigón o concreto con un acabado de piso pulido.

Considere además los costos de demolición, compactación de suelo, desalojo o retiro de ripio (factor de abundamiento) y otros. $\mathrm{El}$ piso puede requerir malla metálica, fibra, endurecedor superficial, epóxico, acelerador de f raguado, lavado y sellado, etc. Evite hacer las construcciones con personal o supervisión no adecuados.

\section{2) Muelles de carga y descarga}

Estas zonas son críticas. Aparte de requerimientos propios de seguridad, los muelles deben permitir el uso de montacargas, grúas y flujos rápidos de carga y descarga.

Las especificaciones técnicas de su diseño pueden variar de empresa a empresa, aún dentro de la 
misma industria Información relevante para su diseño puede encontrarse en las referencias.

En nuestro contexto, uno de los errores más comunes es realizar la operación con cuadrillas y no con instrumentos mecánicos. Muchas veces esto ocurre por no contarse con montacargas y otras por la mala planificación de sus áreas de acceso como ramplas.

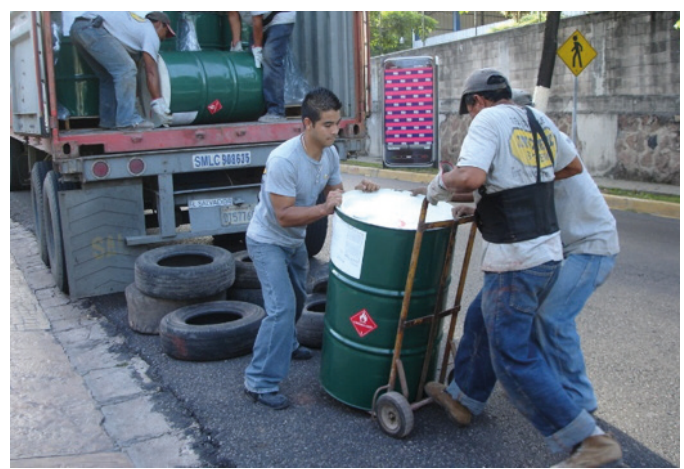

Figura $n .^{\circ}$ 3. Descargo inseguro de materiales peligrosos en plena calle. Fuente: elaboración propia.

\section{3) El layout de las instalaciones}

Las diferentes zonas en las cuales una empresa organiza sus operaciones tendrán un impacto relevante para su productividad. Como buenas prácticas, deben considerarse:

3.1. La integración de todos los factores que afecten la distribución de los productos.

\subsection{La mejora del movimiento de material según} distancias o recorridos mínimos para el picking principalmente.

3.3. Utilización "efectiva" de todo el espacio y volumen del almacén.
3.4. Contribuir al mínimo esfuerzo y seguridad para todos los trabajadores.

3.5. Flexibilidad en la ordenación para facilitar reajustes o ampliaciones.

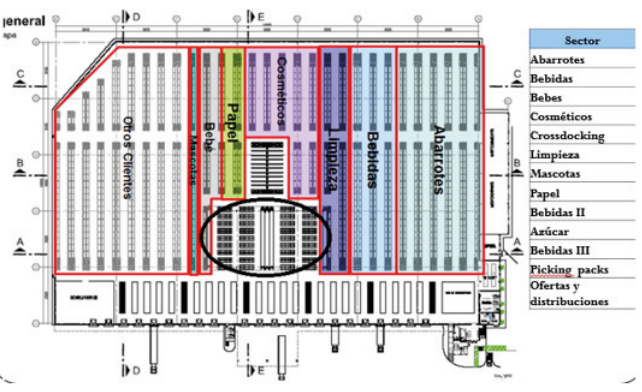

Figura ${ }^{\circ}{ }^{\circ} 4$. Ejemplo de layout de un centro de distribución. Fuente: elaboración propia.

Cada vez más, la organización interna se orienta a mejorar la velocidad, flexibilidad y precisión en la gestión del ciclo del pedido del cliente.

\section{4) Espacios de maniobra}

En la medida de lo posible deben preverse los accesos vehiculares y el tránsito de liste esas unidades de transporte dentro de las instalaciones de la empresa.

La forma en que las empresas diseñan sus áreas de parqueo o maniobras es importante. Puede obstaculizar la secuencia de recepción y despacho de cargas. Los espacios requeridos para parqueo y maniobra son prácticamente dos veces la longitud máxima de las unidades de transporte recibidas. Las bahías a 90 grados proveen espacio para un mayor número de vehículos, pero requieren mayor espacio de maniobra. Las bahías en ángulo, pueden albergar un menor número 
de unidades pero requieren menor espacio de maniobra tal como se ilustra en la Figura n. 5.
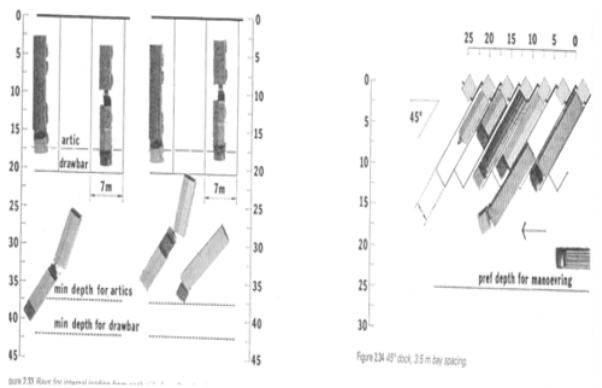

Figura.$^{\circ}$ 5. Ejemplo de Bahías a 90 grados y en ángulo. Fuente: Drury, Jolyon and Falconer Peter. Building and Planning for Industrial Storage and Distribution, Architectural Press, 2a Edición, 2003.

\section{5) Distancia entre pasillos}

Aunque parece obvio, muchas empresas no han sido conscientes de su importancia en los flujos logísticos y han preferido almacenar más producto a expensa de los pasillos. El resultado es que el flujo de productos se atasca, bien en el almacenamiento o bien en su recolección para despacho.

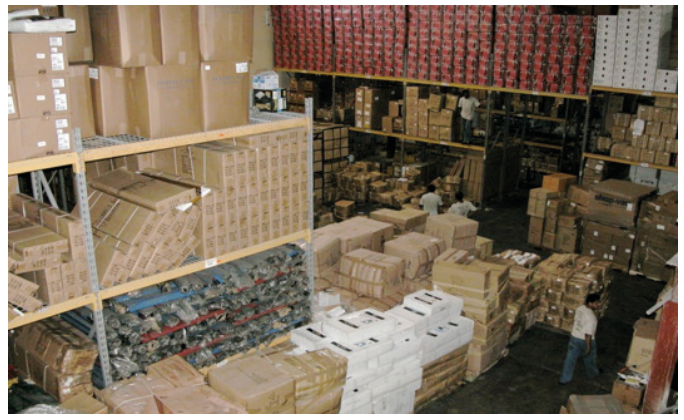

Figura n. ${ }^{\circ}$ 6. Almacenamiento defectuoso con ancho de pasillo insuficiente. Fuente: elaboración propia.
Los pasillos deberán facilitar la colocación y extracción de productos de las estanterías. En función del montacarga a utilizarse, los pasillos deben ser al menos de tres metros. De nada servirá contar con montacargas cuando los pasillos son estrechos, pues los productos deberán reacomodarse manualmente en altura. No se utiliza la unidad logística apropiada (pallet) y todo deberá ser manejado pieza por pieza. Particularmente peligroso en el manejo de cargas a altura. Véase la Figura n. ${ }^{\circ} 6$.

\section{B. Análisis de procesos}

El segundo punto de la metodología IPISI aborda los procesos. Todas las empresas cuentan con procesos, pero no todas tienen procedimientos. $\mathrm{El}$ procedimiento es el proceso escrito. Es importante revisar que las operaciones logísticas estén lo suficientemente documentadas y que sus empleados las realicen como está estipulado, con eficacia y eficiencia. Es común encontrar; sin embargo, que las empresas o instituciones no cuentan con procedimientos actualizados o validados por la gerencia. Curiosamente, algunos procedimientos, especialmente burocráticos, no aportan valor a los clientes, ni se tienen indicadores de desempeño para los mismos. No llevan a ningún lado.

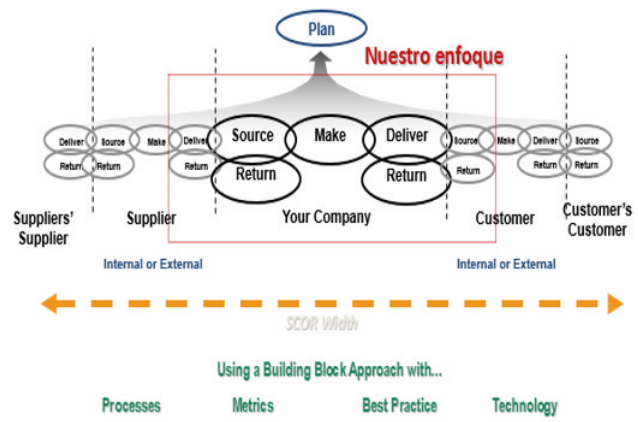

Figura n. ${ }^{\circ}$ 7. Supply Chain Operations Reference SCOR. Fuente: Supply Chain Council. 
A nivel macro es muy ilustrativo el uso del modelo logístico Supply Chain Operations Reference SCOR. Este modelo fue desarrollado e implementado por el Supply Chain Council (SCC) en 1996 con el objetivo de estandarizar la referencia del proceso de la cadena de suministro activado por una comunicación efectiva entre los socios a través del:

- Uso de terminología estándar para mejorar la comunicación y conocer los temas que preocupan la cadena de suministro.

- Uso de métricas o KPI's estándares para medir el desempeño y realizar el benchmarking.

El modelo SCOR está fundamentado sobre tres pilares: modelado de Procesos, Métricas de Desempeño y Mejores Prácticas. En la actualidad, se utiliza la versión 12 soportada por la American Production and Inventory Control Society (Asociación para el Control de la Producción y los Inventarios APICS).

Se debe recordar que los procedimientos son los “ojos del gerente”. El gerente no puede estar en todo, pero sí puede determinar y comunicar la mejor forma que existe dentro de la compañía para hacer las cosas.

Debe revisarse el estado actual de los procedimientos y la calidad de los mismos. Se sugiere analizar los procesos contenidos en el modelo SCOR y compararlos contra los que se ejecutan en la empresa. Encontraremos procesos que no se ejecutan, otros que se comienzan a realizar y otros que están automatizados. Esto es lo que se conoce como madurez del proceso y se ilustra en la Figura n. ${ }^{\circ} 8$.
Modelo de madurez de los

procesos logísticos

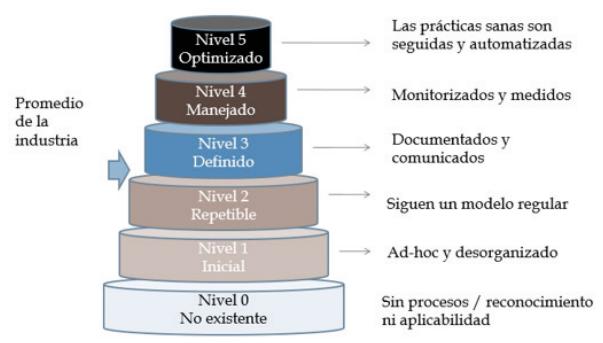

Figura n. ${ }^{\circ}$ 8. Modelo de madurez basado en Capability Maturity Model del Software Engineering Institute SEI de la Universidad Carnegie - Mellon.

Existen muchas maneras de graficar un proceso mediante diagramas de flujo. Normalmente se grafican en el programa MS VISIO pero igualmente puede utilizarse el modelo SIPOC para visualizar los puntos críticos en él. Se sugiere registrarse gratuitamente en la herramienta para diagrama de flujos https://www.lucidchart.com

Crear un mapa de procesos resulta muy importante como una ayuda gráfica que nos permita visualizar el flujo y organización de la información. No solamente se entenderá mejor, sino también se visualizarán las áreas de mejora.

\section{Análisis de inventarios}

El stock es una provisión de artículos en espera de su utilización posterior con el objetivo de disponer del producto correcto, en la cantidad correcta, en el tiempo correcto, en el lugar correcto al mínimo costo.

Aparte de los datos de rotación, cobertura de inventario, inventario muerto, exactitud de inventario, resulta valioso conocer. 
- Cuantía del inventario por categoría o línea de producto.

- Categorías $\mathrm{ABC}$ por rotación, costo, etc.

- Antigüedad y obsolescencia versus rotaciones.

- Exactitud del inventario y toma de inventarios cíclicos.

- Nivel de servicio y quiebres de stock.

- Revisar si las compras están acordes al tiempo de aprovisionamiento y clasificación del inventario.

- Rapidez con la cual se complementan pedidos.

- Tecnología utilizada para su control (wms/ códigos de barras/rfid).

Gracias a la ayuda del software y otras tecnologías disponibles, las empresas cuentan hoy en día con más información relevante sobre el comportamiento de sus inventarios, su demanda y proyecciones. Lo importante siempre es ver cómo se traduce la información en decisiones.

El inventario debe analizarse objetivamente y deben considerarse aspectos como el tiempo de aprovisionamiento, la cobertura en días de las existencias, la estimación de la demanda, los pronósticos, etc. Es importante que la empresa tenga visibilidad sobre los procesos de compra, niveles de inventario y ventas a fin de evitar rupturas de stock y excesos de inventarios.

Se debe comprender bien cuáles ítems serán producidos para stock y cuales contra pedido de los clientes. Ambos sistemas tienen requerimientos distintos.

\section{Seguridad laboral y ocupacional}

La mayor parte de la literatura logística lamentablemente deja por fuera el bienestar del trabajador y se enfoca en la productividad, rentabilidad y control de ciclos de aprovisionamiento, producción, almacenamiento y distribución.

Los centros de distribución, en general, son fuente de peligro para los empleados y no debería descuidarse este tema.

Aunque existe la Ley General de Prevención de Riesgos en los Lugares de Trabajo LGPRLT como única normativa aplicable, esta no es específica a los riesgos que existen en un almacén. De igual forma, las normativas OHSAS 18001, ISO 45001, British Standards BS son sistemas completos de gestión de calidad con certificación internacional más enfocados al manejo documental y metodologías de manejo de riesgos. Su certificación resulta onerosa para la mayoría de las pymes. No obstante, el análisis de riesgos, para ser breve, debe realizarse y partirá en establecer los peligros en el almacén y valorar su probabilidad de ocurrencia y el impacto o consecuencia de su materialización. Esto es resumido en la herramienta conocida como la matriz de riesgos del INSHT de España que se ilustra en la Figura n. ${ }^{\circ} 9$.

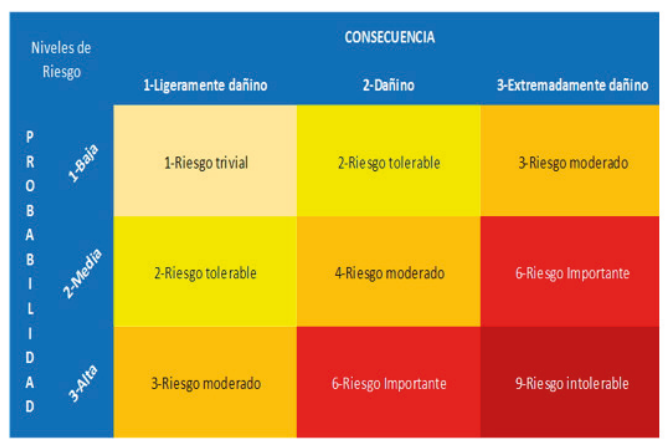

Figura n. ${ }^{\circ}$ 9. Matriz de Riesgos. Fuente: Matriz de riesgos laborales, INHST. Ponencia Jaén Sánchez, 1. a Jornada Técnica de Prevención de Riesgos Laborales, septiembre 2014, España. 
Los riesgos de un centro de distribución con respecto a otro pueden variar en función del tipo de productos que se almacenan, la infraestructura disponible, la idoneidad de los equipos de manejo de materiales, y la incorporación de factores de prevención en las operaciones que se ejecutan.

El tipo de infraestructura y la unidad logística de manejo serán entonces factores críticos en la creación de un entorno seguro para el trabajador. Tómese, por ejemplo, los almacenes que manipulan cargas pesadas como bobinas, metales, producto industrial, carga unitarizada en cajas, pallets o bien contenedores completos.

En ese sentido, el Instituto Nacional de Seguridad e Higiene en el Trabajo de España (NSHT) ha desarrollado Normas Técnicas de Prevención NTP que abarcan actividades muy específicas en las operaciones de un Centro de Distribución y que no se alcanzan a incorporar a las directrices de la LGPRLT de El Salvador. Algunas de ellas son:

- NTP 1112: Seguridad en el almacenamiento de materiales mediante paletizado y apilado sobre suelo - Año 2017.

- NTP 852: Almacenamiento en estanterías metálicas - Año 2009.

- NTP 298: Almacenamiento en estanterías y estructuras - Año 1993.

- NTP 1076: Muelles de carga y descarga: seguridad Año 2013.

- NTP 477: Levantamiento manual de cargas: ecuación del NIOSH - Año 1998.

En cuanto a la seguridad laboral, en los centros de distribución también requieren analizar detenidamente la fuente de riesgos, que para el caso son las instalaciones, las operaciones y el tipo de productos. Los temas de señalización, demarcación, equipo de protección personal y manejo de productos peligrosos deben incluirse.

\section{E. Indicadores logísticos}

La siguiente fase del modelo IPISI son los KPI o Indicadores Logísticos de Desempeño. Resulta clave para cualquier proyecto de mejora contar con medidas de rendimiento cuantificables aplicados a la gestión logística que permiten evaluar el desempeño y el resultado en cada proceso de recepción, almacenamiento, inventarios, despachos, distribución, entregas, facturación y flujos de información entre las partes de la cadena logística. Cada proceso debe medirse al menos con un indicador. Esto permitirá controlar su variabilidad entre valores normales.

Los tipos de indicadores que se encuentran en los centros de distribución pueden ser financieros (costo), de velocidad (tiempo), de productividad, de calidad; y de seguridad y sostenibilidad ambiental.

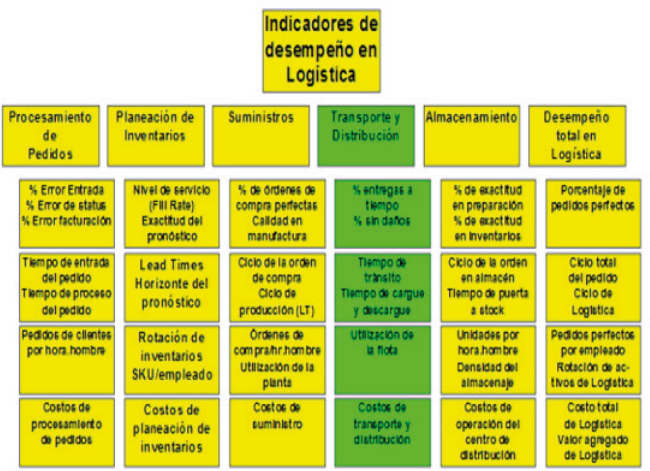

Figura $n .0^{\circ}$ 10. Ejemplo de Indicadores de Desempeño Logístico. Fuente: Vidal, Carlos, Seminario Gerencia de Distribución, DIESCO 2006, San Salvador. 
Existe una gran cantidad de indicadores. Es importante no tratar de abarcar muchos de ellos, sino concentrarse en aquellos que miden "la salud" de las operaciones. Algunos de los más utilizados en el medio son el nivel de servicio, pedidos perfectos, exactitud de inventario, on time in full OTIF, cobertura de inventario, utilización del almacén, etc. Se recomienda consultar el libro Indicadores Logísticos, del Ing. Luis Aníbal Mora, donde se describen ampliamente todos ellos.

Por último, los indicadores deben calcularse regularmente y comparar su evolución en el tiempo. Ello requiere entonces que sean los sistemas informáticos quienes los calculen con base en la información que maneja la empresa. Tratar de calcularlos en forma manual demandará mucho tiempo y no siempre se obtendrá valores exactos. Sin embargo, a veces es lo único que se puede hacer.

\section{Conclusiones}

1. Existen numerosas metodologías para analizar almacenes y centros de distribución que al no ser integrales, no proponen la eliminación de causas raíces del problema de falta de productividad.

2. El modelo IPISI proporciona una secuencia de análisis que favorece un punto de vista holístico.

3. Es indispensable que en todo proyecto de mejora se tenga en mente salvaguardar la integridad de las instalaciones, de los productos y de las personas que allí laboran.

4. Procesos e indicadores logísticos deben estar definidos claramente y, de ser posible, debe buscarse su automatización para garantizar la calidad homogénea en el servicio.

\section{Glosario}

Logística: conjunto de medios y métodos necesarios para llevar a cabo la organización de una empresa, o de un servicio, especialmente de distribución.

Cadena de suministro: se refiere a todos los pasos que toman parte en la preparación y distribución de un producto para su venta.

Almacén: local, edificio o parte de este que sirve para depositar o guardar gran cantidad de artículos, productos o mercancías para su posterior venta, uso o distribución

Centro de distribución: es uno o varios almacenes para carga general o refrigerada, en el cual se reciben, inspeccionan, acondicionan, almacenan, clasifican y se preparan y se envían los productos a tiendas minoristas, al por mayor, o a otros centros de distribución fuera del país o en regiones apartadas.

Metodología: el conjunto de pautas y acciones orientadas a describir un problema

Seguridad laboral: el conjunto de técnicas y procedimientos que tienen por objeto evitar $y$, en su caso, eliminar o minimizar los riesgos que pueden conducir a la materialización de accidentes con ocasión del trabajo,

Outsourcing: la decisión de una organización de entregar una parte de sus procesos, comúnmente en el ámbito logístico, productivo o de negocio 
para que sea desarrollado por una empresa Rubio, J., Villarroel, S. (2012). Seguridad y especializada que, por poseer dicho carácter, se Prevención de Riesgos en el Almacén. Programa asume que puede realizarlo con mayor eficiencia. Aula Mentor. Madrid: Ministerio de Educación de España.

\section{Referencias}

Drury, J., Falconer, P. (2003). Building and Mora,L.(2008). Indicadores de la Gestión Logística, Planning for Industrial Storage and Distribution. 2. ${ }^{a}$ Edición. Nueva York: Architectural Press.

Tompkins, J. (2003). Facilities Planning, 3. a Edición. Nueva Jersey: John Wiley and Sons Inc. 2. a Edición. Medellín: ECOE Ediciones.

Konz, S. (2008). Diseño de Instalaciones Industriales. México: Editorial Limusa. 